\title{
Neurotic disorders and the receipt of psychiatric treatment
}

\author{
P. E. BEBBINGTON, ${ }^{1}$ T. S. BRUGHA, H. MELTZER, R. JENKINS, C. CERESA, \\ M. FARRELL AND G. LEWIS \\ From the Department of Psychiatry and Behavioural Sciences, RF \& UCL Medical School, \\ Institute of Psychiatry and Office for National Statistics, London; Department of Psychiatry, University of \\ Leicester; Ninewells Hospital and Medical School, Dundee; and University of Wales College of Medicine, \\ Department of Psychological Medicine, Cardiff
}

\begin{abstract}
Background. Access to psychiatric treatment by people with neurotic disorders in the general population is likely to be affected both by the severity of disorder and by sociodemographic differences.

Method. In the household component of the National Surveys of Psychiatric Morbidity $>10000$ subjects in Great Britain with psychiatric symptoms were interviewed using the CIS-R. They were also asked about difficulties experienced in performing seven types of everyday activity. All subjects classed as having an ICD-10 disorder were questioned about their experience of treatment with antidepressants, hypnotics, and counselling or psychotherapy.

Results. Less than $14 \%$ of people with current neurotic disorders were receiving treatment for them. Within the previous year, only a third had made contact with their primary care physician for their mental problem: of these $<30 \%$ were receiving treatment. Overall, $9 \%$ of people with disorders were given medication and $8 \%$ counselling or psychotherapy. A diagnosis of depressive episode was that most associated with antidepressant medication. Treatment access was affected by employment status, marital status, and age, but the major determinant was symptom severity. Neither sex nor social class influenced which people received treatment.
\end{abstract}

Conclusions. People with psychiatric disorders seldom receive treatment, even when they have consulted their primary care physician about them. In many cases, this must represent unmet needs with a strong claim on health resources. There are also inequalities in the receipt of treatment, although the major influence is the severity of disorder.

\section{INTRODUCTION}

The need for psychiatric treatment in the whole population is of great importance to potential improvements in public health. 'Need' may be defined as the requirement for treatment as decided by experts in the field (Bradshaw, 1992). Thus, the indications for treatment are decided by people who are clinically trained, even though the actual deployment is increasingly the result

${ }_{1}^{1}$ Address for correspondence: Professor Paul E. Bebbington, RF \& UCL Medical School, Department of Psychiatry and Behavioural Sciences, Archway Campus, Whittington Hospital, Highgate Hill, London N19 5NF. of a process of negotiation between expert and client.

In measuring need we are faced with a dilemma: we can measure it accurately only on samples of a size too small to allow generalization. Bebbington and his colleagues (1997, 1999) have attempted to measure need directly using the community version of the Needs for Care Assessment (Bebbington et al. 1996). However, this was conducted on a relatively small sample and was very time consuming. It is not feasible to devote resources of this magnitude to large scale surveys, despite the fact that we require information of some kind relating to the need for treatment. 
There are ways of measuring need indirectly from other information. In addition to people with a need for treatment, there are several groups relevant to this exercise: those identified as having a psychiatric disorder, those who want ('demand') treatment, those who are assessed by clinicians, and those who are actually receiving treatment. These groups overlap, but not completely. Together, they can provide information illuminating aspects of need, albeit with inevitable reservations.

One of the purposes of the British National Surveys of Psychiatric Morbidity (Jenkins et al. 1997a) was to obtain an indirect evaluation of needs for treatment. The household survey collected information about diagnosis, the severity of disorder, contacts with primary and secondary services and the treatments received by those with a diagnosed disorder. Combining these does allow approximations of the extent to which needs are not being met by services.

Treatment is the second stage of the process of service utilization. The first stage is making contact with an agency that can deliver treatment. In Britain most treatment is provided by primary care physicians. In a previous paper (Bebbington et al. 2000a), we have investigated the influences bearing on whether subjects have made contact with their GPs because of mental problems. In the current paper, we examine how the actual receipt of treatment is associated with symptom severity, social dysfunction and sociodemographic variables.

We expected that only a minority of people diagnosed as cases of psychiatric disorder would receive treatment for them. In this context of limited take-up, one would at least hope that the twin principles of the British National Health Service, of equity and proportionality, would exert an appreciable influence. In other words, a greater proportion of severe disorders would receive treatment than mild ones, and disorders of equivalent severity would be equally likely to be treated whatever the attributes of the person suffering from them. The application of these principles would result in severity being the major determinant of treatment, and the receipt of treatment should not vary in relation to sociodemographic variables. This expectation formed the basis of the predictions that guided our analyses.

Severity has more than one component. We have opted to use as yardsticks of severity the number of psychiatric symptoms, and a measure of difficulties in carrying out everyday activities. In an earlier paper (Bebbington et al. 2000 b), we have shown that these attributes, while correlated, are sufficiently separate to merit using both as independent variables in this sort of investigation. In the current paper we predicted that the major determinant of whether subjects received psychiatric treatments would be the level of psychiatric disturbance, and that sociodemographic variables would be relatively unimportant correlates of treatment.

\section{METHOD}

\section{Sampling}

The methods used in the household survey of psychiatric morbidity have been described in greater detail elsewhere (Jenkins et al. $1997 a, b$; Meltzer et al. $1995 a, b, c)$. The fieldwork was carried out in 1993. The sample was drawn using the small area Postcode Address File as the sampling frame. Two hundred postal sectors covering all of Great Britain, except the Highlands and Islands of Scotland, were selected at random with a probability proportional to the number of delivery points. Within these, ninety delivery points were randomly selected in order to generate a total sample of 18000 delivery points. From among these addresses, private households with at least one person aged 16 to 64 were identified. In all, 15765 private households were identified, and provided 12730 adults eligible for interview, of whom 10108 agreed to take part in the survey. Only one eligible adult (i.e. aged 16-64) was interviewed in each household systematically selected by the Kish grid method (Kish, 1965). Data were weighted to take account of this sampling procedure.

\section{Interviewers and interviewer training}

The interviews were carried out by two hundred interviewers from the Social Survey Division field force of the British Office for Population Censuses and Surveys (now the Office for National Statistics, ONS). These interviewers had a minimum of 3 years' prior interviewing experience, and attended a 1 day training programme in the use of the survey instruments, 
including the CIS-R. Fieldwork was closely monitored by supervisors in the field and by headquarters staff.

\section{Assessment}

Neurotic psychiatric disorder was assessed using the revised Clinical Interview Schedule (CIS-R; Lewis et al. 1992). This has advantages that made it suitable for the National Survey. It can be administered by non-clinically trained interviewers, and training in the use of the schedule was straightforward for the experienced ONS interviewers used in the survey. Moreover, the interview itself is relatively short (on average, $30 \mathrm{~min}$ ) compared with other methods of assessment. CIS-R interviews were carried out only on the 9792 subjects who were interviewed personally. For a variety of reasons, the remaining 316 subjects had to be assessed through interviews with informants.

The CIS-R is made up of 14 sections. Each section covers a particular area of neurotic symptoms, and starts with a variable number of mandatory questions that can be regarded as sift or filter questions. They establish the existence of a particular neurotic symptom in the past month. A positive response to these questions leads the interviewer on to further enquiry giving a more detailed assessment of the symptom in the past week: frequency, duration, severity and time since onset. It is the answers to these questions that determine the informant's score on each section. More frequent and more severe symptoms result in higher scores.

The minimum score for each section is 0 , where it was either not present in the past week or was present only in mild degree. The maximum score is 4 (except for the section on Depressive Ideas, which has a maximum score of 5). Thus, summed scores from all 14 symptom sections range in theory between 0 and 57. The overall threshold score for significant psychiatric morbidity is 12 . In this paper, we have based analyses on a fourfold categorization rather than the total score. This permits the calculation of weighted relative odds for the different levels of the variable. Since data on the receipt of treatment were only obtained from people with a neurotic disorder, this fourfold classification was deliberately skewed towards higher scores $(12-14 ; 15-19 ; 20-24 ; \geqslant 25)$. Note that this is a different categorization from that used in the companion paper (Bebbington et al. 2000a). It was chosen a priori because it gave a reasonable distribution of subjects.

The CIS-R also provides prevalences relating to a 1-week period. Basic symptom data are subjected to a computer algorithm providing ICD-10 diagnoses. All those scoring above 12 on the CIS-R who were not allocated to specific ICD-10 categories were regarded as suffering from mixed anxiety and depressive disorder (F41.2). This ICD-10 category lacks specific diagnostic criteria for research (DCRs). We applied a hierarchy to the diagnostic categories, so that each subject could be allocated to a single primary diagnosis (see Jenkins et al. 1997 b). Subjects who were identified as having a psychotic disorder were not used in our analyses here.

Our method of scoring deficits in activities of daily living (ADL) has been described in detail by Bebbington and his colleagues (2000b). Subjects could score zero, one or two according to whether they had difficulties in seven activities of daily living (personal care, using transport, medical care, household activities, practical activities, dealing with paperwork, managing money), and scores were summed to produce an overall ADL deficit score. The score was collapsed into four categories $(0 ; 1-2 ; 3-4$; $\geqslant 5$ ).

Detailed information was collected from all subjects classed as having an ICD-10 diagnosis about the medication and other forms of treatment they were receiving. The identification of particular classes of drugs was based on the British National Formulary. Thus, antidepressants covered tricyclic antidepressants, mono-amine oxidase inhibitors, compound antidepressants, and other antidepressants, which were mainly selective serotonin reuptake inhibitors (SSRIs). Hypnotics and anxiolytics could also be identified separately. However, for the purposes of the current analyses two broad categories of psychotropic medication were distinguished: antidepressants; and, hypnotics and anxiolytics.

There were two other forms of treatment: psychotherapy and counselling. Although the interviewers sought to distinguish these treatments more precisely, most responses were not very detailed. For this reason the two categories psychotherapy and counselling were 
amalgamated in these analyses. Further details of the definitions of treatment are provided by Meltzer et al. (1995b).

Information about social class, marital status, ethnic origin, employment status and age was obtained from the subjects. In the analyses of social class we excluded those in the armed forces and those who could not be allocated to a group because there was too little information or they had never worked. Marital status categories were collapsed into single; married and cohabiting; and the divorced, widowed and separated. Information about ethnic origin was classified in accord with the official census groupings, but these were then collapsed into a four-fold classification: White; Black (African, African-Caribbean, and 'Black Other'); South Asian (Indian, Pakistani, Bangladeshi); and 'Other'. Employment status also covered four groups: working full-time; working part-time; unemployed; and, economically inactive.

Analyses were based on weighted data. Logistic regression was performed using STATA 6.0 (StataCorp, 1999), which allows the use of data modified by probability weights.

\section{RESULTS}

In Table 1, we display the sorts of treatment that people were receiving according to diagnosis. Remember that for the purpose of this analysis each subject was allocated to a single category according to a hierarchical procedure. This ensured that anyone with co-morbid disorders would be given the diagnosis that we regarded in general as representing the greatest disturbance. Thus, depressive episode in its moderate and severe categories was top of the hierarchy. It can be seen from this table that relatively few patients from any of these groups were receiving psychiatric treatment. In the case of depressive episode, just over a quarter were receiving some form of treatment, while around a fifth were receiving medication. These figures are less for the other diagnostic categories, offering some predictive validity for our choice of hierarchy. Thus, of those with the relatively mild conditions 'mixed anxiety and depressive disorder' and 'generalized anxiety disorder', only $11 \%$ and $8 \%$ respectively were getting treatment. It can be seen that, for people with depressive episode, antidepressants were prescribed more often than anxiolytics. However, this was also true for the diagnoses of phobia and panic, but not for the other conditions. There was thus little evidence of specificity of treatment: the ratio between the prescription of antidepressants and anxiolytics does not vary in the expected way by diagnostic class.

About one-third of the sample with neurotic disorders had contacted their primary care physician for a mental health problem in the past year. As might be expected, the rates for the various treatments in most instances were double what they were in the groups of people with neurotic disorders as a whole. Over forty per cent of the people suffering from depressive episode were in receipt of some kind of treatment, a value actually exceeded by those with phobias. A third of the people who had been to see their GP with a depressive episode were being treated with an antidepressant - which of course still means that two-thirds were not. Thus, contacting a primary care physician did increase the likelihood of potentially effective treatment, but only to levels that remain worrying.

If the presence and type of the neurotic condition does not seem greatly to influence the sort of treatment that these patients are receiving, it may be asked what other influences may change the likelihood. To answer this, we made preliminary analyses of the effect of severity of disorder on access to treatment. The effect of symptom score was striking: people with a symptom score of $<15$ very rarely received psychiatric treatment of any kind $(93 \%$ did not). Every treatment increased gradually in frequency as symptom score increased. Even so, only a quarter of people scoring $\geqslant 25$ on CIS-R were in receipt of any form of psychiatric treatment. Thus, over nearly three-quarters of people in this severe category received no treatment at all. We repeated the analysis using ADL deficit score as our measure of severity. Again, there was an increase in the likelihood of treatment as the score increased. Again, only a minority, even of the most severe group, was in receipt of any form of treatment.

These analyses illustrate two points strongly: first, even among extremely symptomatic people in the general population, psychiatric treatment was given only to a small minority. Secondly, severity, however measured, was a major de- 
Table 1. Type of treatment by type of neurotic disorder in the general population, and in people who had consulted their primary care physician (weighted data)

\begin{tabular}{|c|c|c|c|c|c|c|c|c|c|c|c|c|c|c|}
\hline \multirow[b]{3}{*}{ Type of treatment } & \multicolumn{14}{|c|}{ Type of disorder and percentage of adults with each type of treatment } \\
\hline & \multicolumn{2}{|c|}{$\begin{array}{c}\text { Mixed anxiety } \\
\text { and depressive } \\
\text { disorder }\end{array}$} & \multicolumn{2}{|c|}{$\begin{array}{c}\text { Generalized } \\
\text { anxiety } \\
\text { disorder }\end{array}$} & \multicolumn{2}{|c|}{$\begin{array}{c}\text { Depressive } \\
\text { episode }\end{array}$} & \multicolumn{2}{|c|}{ Phobia } & \multicolumn{2}{|c|}{$\begin{array}{l}\text { Obsessive-- } \\
\text { compulsive } \\
\text { disorder }\end{array}$} & \multicolumn{2}{|c|}{ Panic } & \multicolumn{2}{|c|}{ Total } \\
\hline & pop & pcp & pop & pcp & pop & pcp & pop & pcp & pop & pcp & pop & pcp & gp & pcp \\
\hline Any psychotropic medication & $5 \cdot 6$ & $13 \cdot 5$ & $6 \cdot 0$ & $12 \cdot 5$ & $20 \cdot 9$ & $34 \cdot 7$ & $14 \cdot 3$ & $33 \cdot 3$ & $11 \cdot 9$ & $27 \cdot 5$ & $9 \cdot 9$ & $23 \cdot 1$ & $9 \cdot 0$ & $20 \cdot 3$ \\
\hline Antidepressants & $3 \cdot 3$ & $9 \cdot 1$ & $3 \cdot 0$ & $6 \cdot 8$ & $15 \cdot 1$ & $29 \cdot 2$ & $8 \cdot 7$ & $21 \cdot 4$ & $6 \cdot 8$ & $15 \cdot 7$ & $7 \cdot 5$ & $18 \cdot 5$ & $5 \cdot 7$ & $14 \cdot 6$ \\
\hline Anxiolytics and hypnotics & $2 \cdot 4$ & $4 \cdot 1$ & $3 \cdot 3$ & $6 \cdot 7$ & $8 \cdot 8$ & $10 \cdot 5$ & $5 \cdot 8$ & 11.9 & $7 \cdot 6$ & $17 \cdot 6$ & $2 \cdot 5$ & $3 \cdot 8$ & $4 \cdot 1$ & $7 \cdot 8$ \\
\hline Any counselling or therapy & $6 \cdot 3$ & $12 \cdot 2$ & $5 \cdot 3$ & $9 \cdot 6$ & $13 \cdot 2$ & $18 \cdot 9$ & $14 \cdot 3$ & $31 \cdot 0$ & $10 \cdot 2$ & $17 \cdot 6$ & $5 \cdot 0$ & $15 \cdot 4$ & $7 \cdot 7$ & $15 \cdot 2$ \\
\hline Any treatment & $10 \cdot 6$ & $21 \cdot 6$ & $8 \cdot 3$ & $17 \cdot 3$ & $27 \cdot 8$ & $41 \cdot 7$ & $22 \cdot 1$ & $47 \cdot 6$ & $18 \cdot 6$ & $37 \cdot 3$ & $13 \cdot 6$ & $30 \cdot 8$ & $13 \cdot 9$ & $28 \cdot 5$ \\
\hline Base & 752 & 222 & 302 & 104 & 206 & 96 & 105 & 42 & 118 & 51 & 81 & 26 & 1563 & 541 \\
\hline
\end{tabular}

pop, Type of treatment by type of neurotic disorder in the general population (gp); pcp, type of treatment by type of neurotic disorder in people who had consulted their primary care physician.

Table 2. Logistic regression of treatment by antidepressants, severity of disorder and sociodemographic variables. Model of best fit (weighted data, robust estimates of confidence intervals)

\begin{tabular}{|c|c|c|c|c|}
\hline Factors & Adjusted odds & $95 \% \mathrm{CI}$ & $z$ & $P$ \\
\hline \multicolumn{5}{|l|}{ Symptom severity } \\
\hline $12-14$ & $1 \cdot 00$ & - & - & - \\
\hline $15-19$ & $1 \cdot 48$ & $0 \cdot 7-3 \cdot 0$ & $1 \cdot 06$ & $0 \cdot 29$ \\
\hline $20-24$ & $2 \cdot 27$ & $1 \cdot 1-4 \cdot 6$ & $2 \cdot 28$ & $0 \cdot 023$ \\
\hline$\geqslant 25$ & $4 \cdot 41$ & $2 \cdot 3-8 \cdot 5$ & $4 \cdot 43$ & $0 \cdot 001$ \\
\hline \multicolumn{5}{|l|}{ Marital status } \\
\hline Single & $1 \cdot 00$ & - & - & - \\
\hline $\begin{array}{l}\text { Married or } \\
\text { cohabiting }\end{array}$ & $1 \cdot 35$ & $0 \cdot 7-2 \cdot 8$ & $0 \cdot 81$ & $0 \cdot 42$ \\
\hline $\begin{array}{l}\text { Widowed, separated } \\
\text { or divorced }\end{array}$ & 1.76 & $1 \cdot 1-2 \cdot 8$ & $2 \cdot 34$ & $0 \cdot 019$ \\
\hline \multicolumn{5}{|l|}{ Age, years } \\
\hline $16-24$ & $1 \cdot 00$ & - & - & - \\
\hline $25-34$ & $1 \cdot 84$ & $0 \cdot 6-5 \cdot 2$ & $1 \cdot 14$ & $0 \cdot 26$ \\
\hline $35-44$ & $2 \cdot 81$ & $0 \cdot 9-8 \cdot 5$ & 1.84 & $0 \cdot 005$ \\
\hline $45-54$ & $4 \cdot 52$ & $1 \cdot 5-13 \cdot 6$ & $2 \cdot 69$ & 0.007 \\
\hline $55-64$ & $2 \cdot 75$ & $0 \cdot 9-8 \cdot 5$ & $1 \cdot 76$ & $0 \cdot 079$ \\
\hline \multicolumn{5}{|l|}{ Employment status } \\
\hline Working full-time & $1 \cdot 00$ & - & - & - \\
\hline Working part-time & $1 \cdot 05$ & $0 \cdot 5-2 \cdot 3$ & $0 \cdot 13$ & $0 \cdot 90$ \\
\hline Unemployed & $1 \cdot 43$ & $0 \cdot 7-2 \cdot 9$ & 0.99 & $0 \cdot 32$ \\
\hline Economically inactive & $2 \cdot 62$ & $1 \cdot 4-4.8$ & $3 \cdot 12$ & 0.002 \\
\hline
\end{tabular}

terminant of the likelihood that treatment is given.

We next sought to evaluate the relative contribution of severity of disorder and sociodemographic factors to the likelihood of treatment, in order to test our initial predictions. In Table 2, we present the model of best fit from a
Table 3. Logistic regression of treatment with anxiolytics and hypnotics, severity of disorder and sociodemographic variables. Models of best fit (weighted data, robust estimates of confidence intervals)

\begin{tabular}{|c|c|c|c|c|}
\hline Factors & Adjusted odds & $95 \% \mathrm{CI}$ & $z$ & $P$ \\
\hline \multicolumn{5}{|l|}{ Symptom severity } \\
\hline $12-14$ & $1 \cdot 00$ & - & - & - \\
\hline $15-19$ & $2 \cdot 12$ & $0 \cdot 9-5 \cdot 1$ & 1.68 & 0.093 \\
\hline $20-24$ & $2 \cdot 96$ & $1 \cdot 2-7 \cdot 0$ & $2 \cdot 47$ & 0.014 \\
\hline$\geqslant 25$ & $5 \cdot 82$ & $2 \cdot 6-12 \cdot 9$ & $4 \cdot 35$ & 0.001 \\
\hline \multicolumn{5}{|l|}{ Marital status } \\
\hline Single & $1 \cdot 00$ & - & - & - \\
\hline $\begin{array}{c}\text { Married or } \\
\text { cohabiting }\end{array}$ & 1.92 & $1 \cdot 0-3 \cdot 9$ & 1.83 & 0.067 \\
\hline $\begin{array}{l}\text { Widowed, separated } \\
\text { or divorced }\end{array}$ & $2 \cdot 80$ & $1 \cdot 6-4 \cdot 8$ & $3 \cdot 78$ & $0 \cdot 001$ \\
\hline \multicolumn{5}{|l|}{ Age, years } \\
\hline $16-24$ & $1 \cdot 00$ & - & - & - \\
\hline $25-34$ & $1 \cdot 00$ & $0 \cdot 2-4 \cdot 9$ & $0 \cdot 004$ & $1 \cdot 0$ \\
\hline $35-44$ & $4 \cdot 08$ & $1 \cdot 0-16 \cdot 9$ & 1.94 & 0.052 \\
\hline $45-54$ & $8 \cdot 00$ & $2 \cdot 0-32 \cdot 6$ & $2 \cdot 90$ & 0.004 \\
\hline $55-64$ & $12 \cdot 63$ & $3 \cdot 0-53 \cdot 8$ & $3 \cdot 43$ & 0.001 \\
\hline
\end{tabular}

logistic regression analysis using treatment by antidepressants as the dependent variable. The factors influencing treatment with antidepressants are the number of psychiatric symptoms, marital status, age and employment status. It is clear that by far the strongest influence is that of symptomatic severity, with the most severe category over four times as likely as the least severe to receive antidepressants. With regard to marital status, the least likely to be treated with antidepressants 
Table 4. Logistic regression of treatment by counselling or therapy, severity of disorder and sociodemographic variables. Models of best fit (weighted data, robust estimates of confidence limits)

\begin{tabular}{lcccc}
\hline \hline Factors & Adjusted odds & $95 \% \mathrm{CI}$ & $z$ & $P$ \\
\hline $\begin{array}{l}\text { Symptom severity } \\
\quad 12-14\end{array}$ & $1 \cdot 00$ & - & - & - \\
$\quad 15-19$ & $1 \cdot 47$ & $0 \cdot 8-2 \cdot 8$ & $1 \cdot 16$ & $0 \cdot 25$ \\
$20-24$ & $2 \cdot 92$ & $1 \cdot 6-5 \cdot 4$ & $3 \cdot 42$ & $0 \cdot 001$ \\
$\geqslant 25$ & $2 \cdot 71$ & $1 \cdot 4-5 \cdot 2$ & $2 \cdot 96$ & $0 \cdot 003$ \\
Social impairment & & & & \\
0 & $1 \cdot 00$ & - & - & - \\
$1-2$ & $2 \cdot 71$ & $1 \cdot 4-5 \cdot 1$ & $3 \cdot 12$ & $0 \cdot 002$ \\
$3-4$ & $1 \cdot 29$ & $0 \cdot 7-2 \cdot 5$ & $0 \cdot 75$ & $0 \cdot 46$ \\
$\geqslant 5$ & $2 \cdot 34$ & $1 \cdot 3-4 \cdot 1$ & $2 \cdot 93$ & $0 \cdot 003$ \\
\hline \hline
\end{tabular}

were the single, while the most likely was the divorced, widowed and separated group. The youngest group of our subjects were least likely to receive antidepressants, with those in the age band 45-54 having a relative odds of $>4$. Employment made antidepressant treatment less likely. The economically inactive were over twice as likely to be receiving these drugs. While ethnicity did not add significantly to the model, none of the black or south Asian cases $(N=32$ and 37 respectively) were being prescribed antidepressants and only 2 of the 23 cases of the 'other' group. It is notable that neither ADL deficits nor social class had any effect on antidepressant treatment, but most importantly neither did sex.

We then repeated these analyses for the combined treatment category of anxiolytics and hypnotics (Table 3). The model of best fit required only symptom severity, marital status, ethnic status, and age. Again the number of psychiatric symptoms had a major effect, while the ADL deficit score did not. Increasing age strikingly increased the likelihood of receiving medication of this type, with the oldest group over ten times as likely to receive it than the youngest. Being single reduced the likelihood of being given this sort of drug treatment. It is of interest that no people with neurotic disorders in the non-white groups were in receipt of hypnotics or anxiolytics.

In Table 4, we show the results of a logistic regression using the receipt of counselling or psychotherapy as the dependent variable. The model of best fit was very simple, incorporating only two terms: symptom severity and ADL deficit score. Any deficit at all in activities of daily living appeared to increase the likelihood that counselling would be provided.

\section{DISCUSSION}

In this paper, we have combined information about people in the general population with a diagnosis of neurotic disorder and the treatments they received. We have used this information as a proxy, to draw conclusions about needs for treatment and the extent to which they are unmet. There are potential pitfalls in this process, since diagnosis is only an approximate indication that treatment is needed, and says little about what sort of treatment should be given. There are also problems in the use of the CIS-R to attribute diagnoses, as we ourselves have shown (Brugha et al. 1999). Moreover, we only collected information about treatment from people classed as having a current psychiatric disorder. Thus, we are limited in what we can say about the overall spread of treatment. In particular, we cannot comment on the possible overprovision of treatment in people who do not need it. Our direct assessment of treatment was reasonably detailed, but was based on self-report without corroboration from collateral sources. The approximations inherent in our approach would have made our conclusions dubious, were it not for the fact that the findings were so striking. They are extremely unlikely to have arisen substantially from the imperfections of our information and of the way we have used it.

Thus, these data suggest very strongly that only a small fraction of people diagnosed as having a neurotic disorder in Britain receive anything that could be described as treatment. It is possible that in some cases more detailed psychiatric evaluation of some individuals in our sample might lead to a conclusion that no treatment was required. This could lie behind the very large proportion of people with mixed anxiety and depressive states who are not in receipt of treatment. However, the findings are so dramatic that one must conclude that they represent a large reservoir of untreated psychiatric disorders. Consultation with a primary care physician roughly doubled the chance of receiving all types of psychiatric treatment, but still left over two-thirds of people with depressive 
episode without benefit of an antidepressant. Similar results have recently been reported by Ohayon et al. (1998) in a British telephone survey.

It should be noted that in our study, $6.5 \%$ of subjects who did not report seeing their primary care physician for their mental health problem were nevertheless in receipt of some form of treatment. This is more likely to reflect memory failure than treatment by another route, although in some instances it might indicate longstanding treatment provided through repeat prescription.

In addition to evaluating rates of treatment, our purpose was to identify influences on the receipt of treatment. We studied three separate broad forms of treatment: antidepressants, hypnotics and anxiolytics and psychological treatments, mainly counselling. In no case did sex or social class have an effect on who received treatment, once other clinical and demographic variables had been taken into account. Neither did the presence of physical illness. These results are surprising in themselves. It is clear that the major determinant of who received treatment was symptomatic severity, although this had a somewhat less dramatic effect on the receipt of counselling. Deficits in ADL, which are another measure of clinical severity, had no effect additional to symptom severity except in the case of counselling: people with ADL difficulties were more likely to be referred for counselling.

This impact of severity, with relatively little contribution from key measures of social disadvantage (sex, ethnicity, social class) does suggest that, even in the context of low treatment uptake, the principles of equity and proportionality apply. This result parallels that in our earlier paper on the initial process of making contact with the primary care physician. However, it is of interest that women are more likely to seek consultation with their GP, but once there, are no more likely to be treated for their psychological problems.

There were other clinical and demographic variables that affected access to treatment. Age consistently had an effect. However, the salient age for receiving antidepressants and counselling was 45-54, while the pattern for anxiolytics and hypnotics was for a marked and continuing increase with age. The latter was largely due to the more general use of hypnotics in older populations. Nevertheless, there appeared to be a general trend for younger people to have relatively little access to treatment, and this parallels their reduced likelihood of making clinical contact for a mental health problem in the first place (Bebbington et al. 2000a).

Marital status affected antidepressant and anxiolytic prescribing, with single people having much less access to these treatments. This probably does represent some inequality of provision as there is no reason why the single should have less need after controlling for severity of disorder. On the other hand, marital status had no effect on who received counselling. Although there were relatively few people with neurotic disorders from the non-white groups, it was surprising that virtually none of them were given medication. This may indicate a genuine inequality, although its extent is unclear because of the small numbers.

The reduced access to treatment with antidepressants by those who are in employment may actually reflect reduced need, even after controlling for disorder severity, but it may also indicate that the demands of work prevent attention to health needs.

Very few other studies have attempted to evaluate needs for psychiatric care, and indeed only two are worth describing in the context of the current report. Lehtinen and his colleagues (1990) evaluated the need for treatment in the Mini-Finland Health Survey. Interviewers made judgements about the need for treatment by primary care physicians in cases of less severity. The subjects' own judgements about whether they needed treatment were also recorded. The interviewers reckoned that approximately $9 \%$ of subjects were in need of specialist treatment, whereas only $1.5 \%$ thought so themselves; a further $6 \%$ however, felt that they were 'probably' in need of treatment. This discrepancy may represent a public education gap. Taking all forms of treatment, the authors claim that around $4 \%$ of subjects were receiving adequate treatment, and $14 \%$ showed an unmet need. However, no attempt was made to say exactly what treatment was needed, or by whom it might be provided.

So far there has been only one direct investigation of needs for specific psychiatric treatment and the extent to which they have been met (Bebbington et al. 1997, 1999). This 
was carried in an area of inner south London with high levels of deprivation. A wide range of clinical information was used to rate the community version of the Needs for Care Assessment (NFCAS-C). Ten per cent of the sample were identified as having had a need for the treatment of a psychiatric condition. Less than half of all potentially meetable needs were met, and this was particularly marked for depressive and anxiety states. Thus, as in the current study, a majority of people with mental health problems did not appear to have proper treatment. The authors felt that, given more resources and greater public and medical awareness, most could be treated by family doctors.

These studies cohere with the current report in indicating low rates of treatment for neurotic disorders in the general population, even for the relatively severe condition of depressive episode, diagnosed in only $2 \cdot 1 \%$ of the population in the National Survey (Meltzer et al. 1995a). These disorders are potentially capable of benefiting from treatment, although the evidence for the effectiveness of counselling is not good. We have discussed the implications for the British National Health Service in our earlier paper (Bebbington et al. 2000a). Whatever the response to our results, they inevitably present serious problems for health service planners.

\section{REFERENCES}

Bebbington, P. E., Marsden, L., Brewin, C. \& Lesage, A. (1996). Measuring the need for psychiatric treatment in the general population: the Community Version of the MRC Needs for Care Assessment. Psychological Medicine 26, 229-236.

Bebbington, P. E., Marsden, L. \& Brewin, C. R. (1997). The need for psychiatric treatment in the general population: the Camberwell Needs for Care Survey. Psychological Medicine 27, 821-834.

Bebbington, P. E., Marsden, L. \& Brewin, C. R. (1999). The treatment of psychiatric disorder in the community: report from the Camberwell Needs for Care Survey. Journal of Mental Health $\mathbf{8}$, $7-17$.
Bebbington, P. E., Meltzer, H., Brugha, T., Farrell, M., Jenkins, R., Ceresa, C. \& Lewis, G. $(2000 a)$. Unequal access and unmet need: neurotic disorders and the use of primary care services. Psychological Medicine 30, 1359-1367.

Bebbington, P. E., Brugha, T., Meltzer, H., Farrell, M., Ceresa, C., Jenkins, R. \& Lewis, G. $(2000 b)$. Psychiatric disorder and dysfunction in the UK National Survey of Psychiatric Morbidity. Social Psychiatry and Psychiatric Epidemiology 35, 191-197.

Bradshaw, J. (1972). A taxonomy of social need. In: Problems and Progress in Medical Care, seventh series (ed. G. McLachlan), pp. 71-92. Oxford University Press: London.

Brugha, T. S., Bebbington, P. E., Jenkins, R., Meltzer, H., Taub, N. A., Janas, M. \& Vernon, J. (1999). Cross validation of a general population survey diagnostic interview: a comparison of CIS-R with SCAN ICD-10 diagnostic categories. Psychological Medicine 29, 1029-1042.

Jenkins, R., Lewis, G., Bebbington, P. E., Brugha, T., Farrell, M., Gill, B. \& Meltzer, H. (1997a). The National Psychiatric Morbidity Surveys of Great Britain - initial findings from the Household Survey. Psychological Medicine 27, 775-790.

Jenkins, R., Bebbington, P. E., Brugha, T., Farrell, M., Gill, B., Lewis, G., Meltzer, H. \& Petticrew, M. (1997b). The National Psychiatric Morbidity Surveys of Great Britain - strategy and methods. Psychological Medicine 27, 765-774.

Kish, L. (1965). Survey Sampling. John Wiley \& Sons: London.

Lehtinen, V., Joukamaa, M., Jyrkinen, E., Lahtela, K., Raitasalo, R., Maatela, J. \& Aromaa, A. (1990). Need for mental health services of the adult population in Finland: results from the MiniFinland Health Survey. Acta Psychiatrica Scandinavica 81, 426-431.

Lewis, G., Pelosi, A. J., Araya, R. C. \& Dunn, G. (1992). Measuring psychiatric disorder in the community: a standardized assessment for use by lay-interviewers. Psychological Medicine 22, 465-486.

Meltzer, H., Gill, B., Petticrew, M. \& Hinds, K. (1995a). The Prevalence of Psychiatric Morbidity among Adults Living in Private Households. OPCS Survey of Psychiatric Morbidity in Great Britain. Report 1. HMSO: London.

Meltzer, H., Gill, B., Petticrew, M. \& Hinds, K. (1995b). Physical Complaints, Service Use and Treatment of Adults with Psychiatric Disorders. OPCS Surveys of Psychiatric Morbidity in Great Britain. Report 2. OPCS: London.

Meltzer, H., Gill, B., Petticrew, M. \& Hinds, K. (1995c). Physical Illness, Service Use and Treatment of Adults with Psychiatric Disorders. OPCS Surveys of Psychiatric Morbidity in Great Britain. Report No. 3. OPCS: London.

Ohayon, M., Caulet, M., Priest, R. G. \& Guilleminault, C. (1998). Psychotropic medication consumption patterns in the UK general population. Journal of Clinical Epidemiology 51, 273-283.

StataCorp (1999). Stata Statistical Software, Release 6.0. Stata Corporation: College Station, TX.

Wing, J. K., Mann, S. A., Leff, J. P. \& Nixon, J. N. (1978). The concept of a case in psychiatric population surveys. Psychological Medicine 8, 203-219. 